\title{
ON THE TEAOHING OF ARITHMETIC.
}

REPORT drawn up by the Committee appointed 13th January 1888, and approved by the Society, 8th June 1888.

1. The following report is not a syllabus of arithmetical teaching, far less a formal treatise, but an attempt to reduce to some order the miscellaneous suggestions at the disposal of the committee.* These suggestions are roughly classified into seven groups. Arithmetic includes both a science and an art, or a theory and applications of it to the wants of life. Of the seven divisions in this report the first four have mainly to do with the art, the next two with the science of arithmetic (the division is not observed with thoroughgoing strictness), while the seventh division contains a few general hints for teaching. These divisions may be briefly styled :-

(1.) Terms, (2.) Measures and Constants, (3.) Processes, (4.) Computation, (5.) Theory, (6.) Links, or ideas leading to higher mathematics, and (7.) General Hints.

\section{Terms.}

2. The meaning of certain terms relating to quantity falls to be taught under Arithmetic.

(a) Such as occur frequently, as

Sum, difference, product, quotient, fraction, ratio, reciprocal, prime, odd and even numbers, measure, multiple, power, root, \&c.

Per cent., interest, discount, stock, area, volume, average, \&c.

(b) Such as occur less frequently, as

Index, logarithm, surd, commensurable, \&c.

Specific gravity, velocity, acceleration, work, horse-power.

Of these $(a)$ should be thoroughly mastered, while (b) should be left for advanced pupils, or explained as they occur in problems.

To facilitate the mastery of several terms in $(a)$, the pupil should be made to use, instead of the general word "answer," the particular term appropriate to the case, as sum, difference, G.C.M., price per yard, \&c.

* The Committee was as follows: Dr J. S. Mackay, Messrs A. J. G. Barclay, A. Y. Fraser (Convener), W. J. Macdonald, and Robert Robertson (who was prevented by domestic bereavement from attending the meetings). At the later meetings Mr R. E. Allardice was present by invitation of the Committee. The suggesticns above referred to were made at the December and January meetings, and also by members in communications to the Secretary. 


\section{Mmasurag and Constants.}

3. Of the tables of messures to be committed to memory, the following are recommended as necessary and sufficient :-

\section{Money.}

$$
\begin{aligned}
& 12 \text { pence }(d .)=1 \text { shilling (s. or } /) \\
& 20 \text { shillings }=1 \text { pound }(£ .)
\end{aligned}
$$

\section{Weight.}

16 ounces $(o x)=$.1 pound $(l b$.

112 pounds $\Rightarrow 1$ hundred weight (crot.)

20 hundredweights $=1$ ton.

[7000 grains (gr.) $=1$ pound. 14 pounds $=1$ stone (st.)]

\section{Length.}

12 inches $(i n)=$.1 foot $(f t$.

3 feet $=1$ yard $(y d$.

1760 yards $=1$ mile $(m l$.

[22 yards $=1$ chain $(c h)=$.100 links $(l k$.$) ]$

Surface.

144 or $12 \times 12$ square inches $(s q$. in. $)=1$ square foot (sq.ft.)

9 or $3 \times 3$ square feet $=1$ square yard $(s q . y d$.)

$$
4840 \text { square yards }=1 \text { acre (ac.) }
$$

640 acres $=1$ square mile (oq. ml.)

[ 40 poles $(p l)=$.1 rood (ro.) 4 roods $=1$ acre $=10$ square ohains.]

\section{Solidity.}

1728 or $12 \times 12 \times 12$ cubic inches (c. in.) $=1$ cubic foot (c. ft.)

27 or $3 \times 3 \times 3$ cubic feet $\quad=1$ cubic yard (c. $y d$. $)$

Liquid Measure.

4 gills $=1$ pint $(p t$.)

2 pints $=1$ quart $(q t$.

4 quarts $=1$ gallon (gal.)
Dry Measure.

4 pecks $(p k$. $)=1$ bushel (bus.)

8 bushels $=1$ quarter $(q r$.

\section{[2 gallons-1 pook.]}

\section{Time.}

$$
\begin{aligned}
60 \text { seconds }(\text { sec. }) & =1 \text { minute }(\text { min. }) \\
60 \text { minutes } & =1 \text { hour }(h r .) \\
24 \text { hours } & =1 \text { day. } \\
365 \text { days } & =1 \text { common year }(y r .) \\
366 \text { days } & =1 \text { leap year. }
\end{aligned}
$$


In the foregoing tables the standard units are printed in italics. Fractions are not used.

Other tables to be retained for reference nerely are Apothecary's weight, Troy weight, angular measure, foreign measures, \&c.

4. Oonstants of frequent occurrence should be remembered, such as the circumference of a circle is 3.1416 times its diameter nearly, the circumference of the Earth is 25000 miles nearly, 1 cubic foot of water weighs 1000 ounces nearly, \&c.

Others of less frequent occurrence should be tabulated for reference, as, specific gravities of common substances, iron, lead, silver, gold, mercury, \&c. ; one gallon of water weighs $10 \mathrm{lb}$., and occupies 277.274 cubic inches nearly; fathom, knot; speed of light, of sound, of falling bodies, atmosphere as a unit of pressure, \&c.

\section{Processers.}

5. It should be clearly understood that the fundamental operations of arithmetic are few. From addition and subtraction are developed multiplication and division, involution and evolution; and the numerous "Rules" in text-books are for the most part classified lists of examples of the application of these operations. As a matter of fact, the fundamenlal laws of arithmetic and algebra together are only three in number (see $\S 28$ ).

6. Decimals. - It should be shown that the decimal notation for fractions (that is "Decimal Fractions") is merely an extension of the ordinary notation for integers.

7. Metric System.-The metric system should be taught, even if the time at the teacher's disposal is so small as to compel him to restrict his treatment to a single measure, for example, length. The principle of the system is too valuable to be passed over.

8. Practice. - The old rule of Practice should be omitted. In the cases where a simple fraction will suffice, retain such exercises as, to find the value of a number of articles whose price is a simple fraction of a pound, of a shilling, dc. These exercises can be introduced as applications of fractions. 
9. The Unitary Method and Proportion.-Opinion is by no means unanimous as to the relative values of these two processes. There seems to be no doubt that the unitary method can be used with intelligence at an earlier age than that of proportion, but it should not supplant proportion throughout. The ideas involved in proportion are of more general importance than anything in the unitary method.

10. Evolution.-'The extraction of square root should be taught as a part of arithmetic, because the need for it arises so frequently ; the extraction of cube root should be treated as a part of algebra.

The following are useful approximations, especially the first two.

$$
\begin{aligned}
& \sqrt{ } 2=\sqrt{(50 / 25)}=\sqrt{(49 / 25)} \text { nearly }=7 / 5 \text { nearly. } \\
& \sqrt{ } 3=\sqrt{ }(48 / 16)=\sqrt{ }(49 / 16) \quad " \quad=7 / 4 \quad, \\
& \sqrt{ } 5=\sqrt{ }(80 / 16)=\sqrt{ }(81 / 16) \quad "=9 / 4, " \\
& \sqrt{ } 7=\sqrt{ }(63 / 9)=\sqrt{ }(64 / 9) \quad "=8 / 3 " \\
& \sqrt{ } 11=\sqrt{ }(99 / 9)=\sqrt{ }(100 / 9) \quad,=10 / 3,
\end{aligned}
$$
$18 / 6,19 / 6,20 / 6,21 / 6$.]

\section{Compdtation.}

11. Systematic endeavour should be made to develope in the pupil the power of accurate and rapid computation. This accomplishment can be attained by continued practice. It requires no severer mental strain than does the acquirement of dexterity in any mechanical exercise.

12. Beginners should be taught to compute in such a way that there may, if possible, be nothing to unlearn. "The act of addition must be made in the mind without assistance; you must not permit yourself to say, 4 and 7 are 11,11 and 7 are 18 , \&c., but only 4,11 , 18, \&c." "Learn the multiplication table so well as to name the product the instant the factors are seen; that is, until 8 and 7 , or 7 and 8 , suggest 56 at once, without the necessity of saying ' 7 times 8 are 56.'" (De Morgan's Arithmetic, 5th edition, pp. 162, 163.)

13. The addition table should be committed to memory as strictly as the multiplication table-both having been first of all constructed by the pupil, with the aid of counters or the like.

14. There should be systematic practice at exercises containing all the typical difficulties (compare the pianist's practice to acquire 
technical dexterity.) For exercises of this kind see De Morgan (1) Arithmetic, 5th ed., appendix, (2) Supplement to Penny Cyclopadia, article Computation, (3) British Almanac and Companion for 1844.

15. As a further aid to rapid computation, the pupil should be exercised in certain labour-saving devices, namely, (1) methods to meet particular frequently-occurring cases (see $\$ 16$ following), and (2) the general abbreviated methods of working to produce results to any required degree of approximation (see $\$ 18,19$ ).

16. Short and easy methods. - It is not suggested that all the methods in the following list (which does not pretend to be exhaustive) should be systematically taught.

Addition.-Example.-In adding two numbers, the first or second may be increased, and the second or tirst diminished to the same extent.

As,

Or again,

$$
\begin{aligned}
57+39 & =60+36 . \\
\text { or } & =56+40 . \\
57+39 & =57+30+9 . \\
\text { or } & =50+39+7 .
\end{aligned}
$$

Subtraction.-Example.-In subtracting two numbers, both may be equally increased or diminished.

As,

$$
\begin{aligned}
57-39 & =58-40 \\
346-328 & =46-28
\end{aligned}
$$

The following is frequently useful :

$$
100-58 \cdot 2476=41 \cdot 7524 \text {. }
$$

Subtract, 5 from 9, 8 from 9, 2 from $9, \&$ c., 6 from 10.

Multiplication.-When the multiplier ends with 5 , in general double it, and halve the answer, or, when the multiplicand is even, halve the multiplicand before multiplying.

To multiply by

multiply by

and divide by

To multiply by

multiply by

$$
\begin{aligned}
& 5,5^{2}, 5^{3}, 5^{4}, \& \mathrm{c} . \\
& 10,10^{2}, 10^{3}, 10^{4}, \& \mathrm{c} . \\
& 2,22^{2}, 2^{3}, 2^{4} \text {, \&c. } \\
& 9,99,999, \text { dc. } \\
& 10,100,1000, \& \mathrm{c} .
\end{aligned}
$$

and subtract the multiplicand. No working need be written down, only the answer.

To multiply by $11,111,1111$, \&c. If by 11 , suppose a zero to be placed to right and left of the multiplicand. Beginning from right, add the digits in successive pairs. If by 111 , suppose two zeros so placed, and add in successive threes, drc. 
To multiply by $98,97,96$, dc., multiply by 100 , and subtract $2,3,4$, dce., times the multiplicand. Similarly for 998, 997, \&o.

To multiply by $42,63,189$, \&c., use the first line of the partial product instead of the multiplicand to get the second line.

To square a number of two digits. The identity $a^{2}=(a-b)(a+b)+b^{2}$, gives for example $58^{2}=56 \times 60+2^{2}$.

Division.-When the divisor ends in 5 , in general double both divisor and dividend.

To divide by multiply by and divide by

$$
\begin{array}{r}
5, \quad 5^{2}, 5^{3}, 5^{4}, \text { \&c. } \\
2, \quad 2^{2}, 2^{2}, 2^{4} \text {, \&c. } \\
10,10^{2}, 10^{3}, 10^{4} \text {, \&c. }
\end{array}
$$

To find the value of 12 articles, given the price of one. Consider the pence in the price as shillings.

To find the value of 20 articles, given the price of one. Consider the shillings in the price as pounds.

To find the value of 100 articles, given the price of one. For every farthing take as many pence and twice as many shillings.

To find the price of 112 pounds, given the price of one pound. Add the price of 12 to the price of 100 .

Percentage.-To find 5\% of a sum of money, take ls. for every $£ 1$ in the sum. For $2 \frac{1}{2} \%$ take 6 d. for every $£ 1$.

Five per cent. per annum is a penny per pound per month. Similarly for two and a half per cent.

17. Practice in the approximate methods should be kept up from the earliest acquaintance with the extended decimal notation. The young pupil, instead of being pushed through the weary and unprofitable mazes of circulating decimals, should rather be exercised in the metric system, in approximate addition, subtraction, multiplication, and division, and in learning to turn the decimal system to account in treating questions of British money.

18. The abbreviated methods of multiplication and division are now given in all good text books, and therefore need not be treated in detail here. The following recommendations are made.

a. In contracted multiplication the multiplier need not be written reversed. This procedure, though a useful and obvious expedient to the mature judgment, sometimes puzzles beginners. The pupil should be accustomed to multiply, beginning at the left as well 
as at the right, and if the units, tens ... digits of the multiplier be placed under the units, tens ... digits of the multiplicand, the pupil easily learns to take the figures of the multiplier in any order whatever, and to place the product correctly. When this point has been reached, he may be allowed to carry on multiplication from the right in the usual way, and then by applying the principle involved in the statement that "a 3rd place decimal, multiplied by a 2nd place decimal, gives a 5th place decimal," he readily learns where he should begin to multiply in order to obtain the requisite approximation.

(b) In performing any long division the pupil should be required to get the successive remainders without writing down the partial products. This is quite easy, even without the special preparation recommended in $\S 14$.

Thus the procedure to get the last remainder in the annexed example, stated in its fullest form for the sake of illustration, is :4 times 7,28 and 9 (write 9 down) 37 , carry $3 ; 4$ times 5,20 , and 3 carried, 23 and 1 (write 1 down) 24, carry 2 ; 4 times 3,12 and 2 carried, 14, and 2 (write 2 down) 16.

$357) 83757(234$

1235

1647

219

19. To express shillings, pence, and farthings as the decimal of a pound, note that

$$
\begin{aligned}
& 2 /=\cdot 1 \quad \text { of } £ 1 . \\
& 1 /=\cdot 05 \text { of } £ 1 . \\
& \frac{1 d}{d}=\cdot 001+1 / 24 \text { of } \cdot 001 \text { of } £ 1 .
\end{aligned}
$$

Hence the following rule, which gives the result correct to the thousandth of a $£$ : Call the florins tenths, the odd shilling (if any) five hundredths; for the thousandths reduce the rest to farthings, adding 1 if there be more than 12,2 if more than 36 .

$$
\text { Examples : (1) } \begin{aligned}
£ 2\|7\| 1 \frac{3}{4} & \\
3 \mathrm{fl} . & =\cdot 3 \\
1 \mathrm{~s} . & =\cdot 05 \\
7 \mathrm{f} . & =\cdot 007
\end{aligned}
$$


(2)

$$
\begin{aligned}
£ 3 \text { " } 16 \text { " } 5 \frac{1}{4} & \\
8 \mathrm{fl} . & =\cdot 8 \\
21 \mathrm{f} . & =022
\end{aligned}
$$

$£ 3 \cdot 822$.

$$
\begin{aligned}
£ 4 \text { " } 15 \text { " } 1014 & \\
7 \text { fl. } & =\cdot 7 \\
1 \mathrm{~s} . & =\cdot 05 \\
41 \mathrm{f} . & =\cdot 043
\end{aligned}
$$

$£ 4 \cdot 793$.

The inverse operation is as follows:-

$$
\begin{aligned}
£ 7 \cdot 293 & =£ 7 \cdot 2+05+\cdot 043 . \\
& =£ 7 " 4 /+1 /+41 f . \\
& =£ 7 " 5 " 10 \frac{1}{4} . \\
£ 4.567 & =£ 4 \cdot 5+\cdot 05+\cdot 017 . \\
& =£ 4 " 11 " 4 .
\end{aligned}
$$

Verifiable exercises on the above can be got at once from the ordinary examples of the compound rules. Turn a column of a compound addition sum into decimals, add the lines as decimals, convert the answer into $\mathfrak{f} \mathbf{s}$. d., and compare the result with that obtained in the usual way. Similarly with subtraction, multiplication, and division. The pupil will see how the absolute error increases in multiplication and diminishes in division, the relative error remaining the same.

20. Logarithms.-The last matter requiring discussion under the head of computation is the question of teaching logarithms.

It is recommended that a knowledge of logarithms be considered a necessary part of a complete arithmetical training. The use of logarithms with a sufficient amount of theory can be taught without the aid of advanced algebra.

\section{Theory.}

21. The necessity of developing the theory of arithmetic, in immediate connection with the practice of the special processes is universally acknowledged, but there is some difficulty in effecting this. A few suggestions are offered on points where a knowledge of theory is most frequently found wanting. 
22. To recommend any text-books in use in this country would be invidious and perhaps useless, but such objection does not apply to foreign treatises. The two following may be mentioned as affording instances of the adaptation to arithmetic of theory which is usually left to algebra:-

(1.) Traité Elémentaire d'Arithmétique, par J. F. J. Kleyer [part 1 ( 75 centimes), part 2 (75 centimes), part 3 ( $1 \cdot 10$ francs)]. H. Dessain, Liége, rue Trappe, 7. 1885-6.

(2.) Traité d'Arithmétique Elémentaire, par L'Abbé E. Gelin (5 francs), Paris, Librairie du Journal de Mathématiques Élémentaires, rue des Écoles, 17. 1886.

23. Scales of Notation.-The theory of other scales of notation may be introduced to illustrate the decimal scale, if only to show the pupil that there is nothing essential in the selection of 10 as the radix of the ordinary scale. The Roman notation should be explained, and examples given in writing and reading it.

24. Divisibility.-The tests of divisibility of numbers by 2,3 , \&c., should be given and proved.

25. Greatest Common Measure.-The theory of the method of finding the G. C. M. by reduction to primes, and of the ordinary method, should be explained.

This last may be done by means of the two following theorems, of which proofs may be given that are perfectly general in method, although particular numbers may be employed.

a. Every common measure of two numbers, say 36 and 28 , is a measure of their difference 8 (and also of their sum 64).

Thus every common measure of 36 and 28 is a common measure of 28 and 8 .

$b$. Conversely, every common measure of the less of two numbers 28 and their difference 8 is a measure of the greater of the numbers 36 .

Thus any common measure of 28 and 8 is a common measure of 36 and 28.

From these two theorems it follows that the problem of finding the G. C. M. of the numbers 36 and 28 may be reduced to the simpler problem of finding the G. O. M. of the numbers 28 and 8 , and this again to that of finding the G. C. M. of 20 and 8 , and so on till the G. O. M. is found. 
Moreover, instead of taking the difference of, say, 28 and 8 , then the difference of 20 and 8 , and again the difference of 12 and 8 , we may take the difference of 28 and any multiple of 8 , for instance, the difference of 28 and $8 \times 3$ or the difference of 28 and $8 \times 4$.

26. Proportion.-The following is given as a very brief outline, sufficient for arithmetic, of the theory of proportion. In the proofs letters are used, but they are meant to be given alongside of full numerical exemplification (see $\S 29$ ).

Definition 1.-The ratio of one whole number to another is measured by the fraction which the one is of the other; and the ratio of one quantity to another is the ratio of the two whole numbers that express these quantities in terms of the sarne unit.

Definition 2.-When two ratios are equal, the four quantities form a proportion.

Theorem 1.-If the fourth term of a proportion be greater than the third, the second shall be greater than the first, if equal equal, and if less less.

Let $\quad a: b=c: d$ be the proportion.

Because $a: b=c: d$,

therefore $\quad \frac{a}{b}=\frac{c}{d}$.

Now (1) if $d$ be greater than $c$,

then the fraction $\frac{c}{d}$ is less than 1 ;

therefore the fraction $\frac{a}{b}$ is less than 1 ;

therefore $b$ is greater than $a$.

(2) If $d$ be equal to $c$,

then the fraction $\frac{c}{d}$ is equal to 1 ;

therefore the fraction $\frac{a}{b}$ is equal to 1 ;

therefore $b$ is equal to $a$.

(3) If $d$ be less than $c$,

then the fraction $\frac{c}{d}$ is greater than 1 ;

therefore the fraction $\frac{a}{b}$ is greater than 1 ;

therefore $b$ is less than $a$. 
Theorem 2.-In a proportion the product of the first and fourth terms is equal to the product of the second and third.

Let

$a: b=c: d$ be the proportion.

Because

$a: b=c: d$,

therefore

$$
\frac{a}{b}=\frac{c}{d}
$$

therefore

$$
\frac{a}{b} \times b \times d=\frac{c}{d} \times d \times b \text {; }
$$

therefore

$$
a \times d=b \times c \text {. }
$$

Application-First.-Theorem 1 enables us to arrange in a proper order any four quantities among which a proportion is assumed to exist.

Example.-If $2 \mathrm{lb}$. of tea cost 5/, what will $6 \mathrm{lb}$. cost?

It is assumed that the ratio of $2 \mathrm{lb}$. to $6 \mathrm{lb}$. is equal to the ratio of the price of $2 \mathrm{lb}$. to the price of $6 \mathrm{lb}$.

Let $x /$ denote the unknown price, which it is usual to write as the fourth term of the proportion. Then the third term will be 5/, for a ratio can exist only between quantities of the same kind.

To ascertain whether $2 \mathrm{lb}$. or $6 \mathrm{lb}$. should occupy the second place, we inquire whether $x$ / is greater than 5/, equal to it, or less than it. On consideration of the question it will be seen that $x /$ must be greater than $5 /$, since it is the price of a larger quantity; that is, the fourth term of the proportion is greater than the third. Hence the second term must be greater than the first; therefore $6 \mathrm{lb}$. must be the second term, and $2 \mathrm{lb}$. the first.

Second.-Theorem 2 enables us to supply any term of a proportion which may be omitted.

Example.-In the proportion $2 \mathrm{lb}: 6 \mathrm{lb} .=5 /: x /$, supply the omitted term.

The proportion may be written therefore therefore

$$
\begin{aligned}
2 \mathrm{lb} .6 \mathrm{lb} & =5 /: x / \\
2: 6 & =5: x \\
2 \times x & =6 \times 5 \\
x & =\frac{6 \times 5}{2}=15 .
\end{aligned}
$$

Hence the omitted term is $15 \%$. 


\section{LINKS.}

27. The recommendations under this heading refer more to the spirit than to the substance of the teaching of arithmetic, and definite suggestions must be few. The teacher of arithmetic who knows, at least, algebra and geometry, and is not tied down to the notion that things with different names must be kept severely apart, no matter how close their relation, can always be trusted to teach arithmetic so as to make prominent those general ideas which, though fully developed only in the higher branches of mathematics, are already of fundamental importance in elementary arithmetic.

28. The Three Fundamental Laws.-In any attempt to deal with arithmetic as a science, an endeavour should be made to show the pervading importance of the laws of commutation, association, and distribution. With a little skill in the introduction of these notions and a word in season to keep them alive, it is by no means difficult to obtain satisfactory results even with young pupils. The pupils should gain familiarity with these laws as exemplified in addition, subtraction, multiplication, and division. At every stage of an example (say of reducing a particular fractional expression) they should occasionally be required to refer the process to the fundamental law under which it comes.

The development of the science of algebra from these laws has been carried out in the best recent text books, which may be consulted for full details; while the same has been done for arithmetic by Homersham Cox (Principles of Arithmetic, Deighton, Bell \& Co., Cambridge, 1885).

29. Generalised Examples.-It is recommended that examples in arithmetic should be systematically generalised. Thus, following a series of such examples as: If $2 \mathrm{lb}$. of tea cost 5/ what will $6 \mathrm{lb}$. cost $?$ there should be given such as: If $p \mathrm{lb}$. of tea cost $q$ / what will $r$ lb. cost? and then it should be shown how the answer to such a general question contains as particular cases the results of all numerical examples of the same class. Work of this kind ensures the intelligent use of the formulae commonly used in problems of interest and the like. 
30. Mensuration.-Thoroughly practical examples in mensuration, derived from actual measurement of the school surroundings, with the making of actual plans, can be made a sound basis for geometry, when the pupils come to study it. Whether arithmetical illustrations be part of the teaching of geometry, or geometrical notions be drawn on for examples in arithmetic, is immaterial ; but the two subjects should certainily be made to lend mutual aid, as here suggested.

\section{Gmanal Hivts.}

31. Do not hurry from stage to stage in arithmetic at the sacrifice of thoroughness. It is not necessary that a pupil of thirteen should be familiar with stocks and shares.

32. Give many easy examples illustrating principles at the introduction of every new process. This is the best kind of "mental arithmetic."

33. In problems involving a number of multiplications and divisions, insist on having all the steps indicated before any "working out" is attempted. In most cases this leads to great simplification.

34. Encourage the pupil always to make a rough forecast of the result. (To originate this habit it will be found a help to make the pupil write his rough estimate on one side of his slate before proceeding to find the exact result on the other.) The habit of doing this is beneficial in two ways :-

a. It shows the importance of approximate methods generally, and in particular of beginning in multiplication at the left of the multiplier.

b. It will prevent the pupil from resting content with a ridiculous answer.

35. Some method of encouraging speed should be adopted, such as numbering or rearranging the pupils as they finish working. The names of the first half dozen written on the blackboard, even if no additional marks be allowed them, stimulates activity. This plan should, of course, be adopted only at intervals. 
36. An attempt should be made to give the pupils some idea of the magnitude of the units of length, weight, \&c. Thus, diagrams of the units of the metric system should be placed on the walls of the schoolroom; measurements of the classroom, blackboards, dc., might be made and recorded where they can be readily seen. The weights and dimensions of the slates and the text-books might be ascertained.

37. In practical applications of arithmetic (to problems of money, \&c.) some care should be taken that the problems are such as actually do occur. For example, it should be borne in mind that income-tax is never calculated on odd shillings, that the Post Office Savings Bank does not allow interest for a fraction of a month, that banks do not deal in fractions of a penny, and so on.

In teaching "Stocks" the money column of the newspaper should be explained, and be made to furnish problems. It may be mentioned that share lists giving particulars (amount paid up per share, \&c.) not contained in the newspapers, can be procured from stockbrokers, or from banks.

For other practical problems geometry and physics should be drawn upon as largely as possible.

38. An operation or a notion should be taught along with its inverse, as, addition with subtraction, multiplication with division, power with root, measure with multiple, dc.

39. As far as exigencies of examinations will allow, postpone formal algebra. The present tendency is to begin algebra too soon by a year or two. 